\title{
Über die Freilandkultur von Taiwania in Deutschland
}

\author{
T. Pauls, M. Radscheit, H. Nimsch, S. Giefer, W. Lobin \& W. Barthlott
}

\begin{abstract}
Taiwania cryptomerioides HAYATA (Cupressaceae, formerly Taxodiaceae) is an attractive monotypic conifer from China, Taiwan, Myanmar and North Vietnam growing up to $60 \mathrm{~m}$. It was discovered in 1904 and since the 1920 s it is in cultivation in temperate houses in Europe (England).

We were able to demonstrate its successful cultivation in areas of Northwest Germany (Bonn) and Southwest Germany (Freiburg) where the species is successfully kept outdoors for more than 20 years. Taiwania cryptomerioides is a new attractive addition for parcs and arboreta, especially for the out of door cultivation in temperate zones Europe and North-America.
\end{abstract}

\section{Zusammenfassung}

Taiwania cryptomerioides HAYATA (Cupressaceae - ehem. Taxodiaceae) ist eine attraktive monotypische bis $60 \mathrm{~m}$ hohe Konifere aus China, Taiwan, Myanmar und Nord-Vietnam. Erst 1904 entdeckt, ist sie seit den 1920er Jahren in den Kalthäusern in Europa (England) in Kultur. Wir konnten an mehreren Standorten in Nordwest-Deutschland (Bonn) und Südwest-Deutschland (Freiburg) aufzeigen, dass die Pflanze teilweise seit über 20 Jahren im Freiland erfolgreich kultiviert wird. Taiwania cryptomerioides ist eine neue attraktive Bereicherung für Parks und Arboreten, geeignet für die Freilandkultur im temperaten Europa und Nordamerika.

\section{Einführung}

Die 1906 beschriebene Taiwania cryptomerioides HAYATA aus der Familie der Cupressaceae hat ein disjunktes, reliktartiges Areal in SW-China (Yunnan und Xizang), Zentral-Taiwan, in den Bergketten NO-Myanmars (ehem. Burma) sowie im Norden Vietnams. Das kleine Vorkommen in den Hoang Lien Mountains in Nordvietnam wurde erst 2002 entdeckt (FARJON 2005).

Fossile Taiwania-Arten findet man im Tertiär von West-Europa bis Japan im gesamten Gebiet der Paläarktis, in Nord-Amerika scheint sie zu fehlen. Wie die meisten früher den Taxodiaceae zugeordneten Gattungen handelt es sich auch bei Taiwania um eine reliktäre monotypische Gattung, die zeitweise sogar als eigene Familie Taiwaniaceae HAYATA geführt wurde.

Taiwania cryptomerioides ist eine Pflanze der Gebirge, die in Höhenlagen zwischen 500 2800 m (China) vorkommt. Die jährlichen Niederschläge sind hoch und können $4000 \mathrm{~mm}$ übersteigen. Nach FARJON (2008) kann Taiwania möglicherweise 2000 Jahren alt werden. Die größten gemessenen Exemplare erreichen bei einem Stammdurchmesser von über $3 \mathrm{~m}$ eine Höhe von $65 \mathrm{~m}$.

Taiwania cryptomerioides wurde um 1920 durch E. WILSON in England und Nord-Amerika eingeführt und befand sich zunächst nur in
Gewächshaus-Kultur (TAROUCA \& SCHNEIDER 1923). Erste Auspflanzversuche erfolgten 1925 im Bedgebury-Arboretum südöstlich von London (DAlimore 1937). Nach Deutschland wurde sie wohl erst nach dem Zweiten Weltkrieg eingeführt, genauere Unterlagen hierzu fehlen allerdings. In dem kürzlich erschienenen, äußerst interessanten Buch von GRIMSHAW \& BAYTON (2009) über neue Bäume in der Freiland-Kultur ist Taiwania cryptomerioides erstaunlicherweise nicht $\mathrm{zu}$ finden.

\section{Freilandkultur von Taiwania in Deutschland}

Taiwania cryptomerioides galt in Deutschland als prinzipiell nicht winterhart. Die Art wurde nur in Kalthäusern kultiviert (z.B. ENCKE 1958). Die ersten Versuche, Taiwania cryptomerioides im Freiland auszupflanzen, wurden wohl im Arboretum des Forstamtes Freiburg-Günterstal unternommen. In dem am Rande des stärker kontinental beeinflussten Schwarzwaldes in Südwestdeutschland gelegenen Arboretums wurden insgesamt fünf Sämlinge in drei aufeinander folgenden Jahren gepflanzt: zwei 1988, zwei 1989 und einer 1990. Alle Pflanzen sind bis heute gesund und haben bei einem Alter von etwa 25 Jahren eine Höhe bis zu 11 m erreicht. Das Gelände liegt auf $350 \mathrm{~m} \mathrm{NN}$ in einem Gebiet 
sehr hoher Jahresniederschläge (1000 mm). Der Tiefstwert der Temperatur lag zwischen 1989 und 2009 bei minus $15^{\circ} \mathrm{C}$. Eine Freilandkultur ist problemlos möglich.

Weitere erfolgreiche Freiland-Auspflanzungen in Deutschland folgten u. a. in den Botanischen Gärten der Universität Bonn, im Botanischen Garten der Universität Bochum (DöRKEN \& HöggemeIer 2009) und bei einigen Privatpersonen.

In den Botanischen Gärten der Universität Bonn wurde Taiwania seit Jahrzehnten im Kalthaus kultiviert. Ein 8-jähriges, etwa $90 \mathrm{~cm}$ großes Exemplar wurde im Frühjahr 2004 an einer ab-

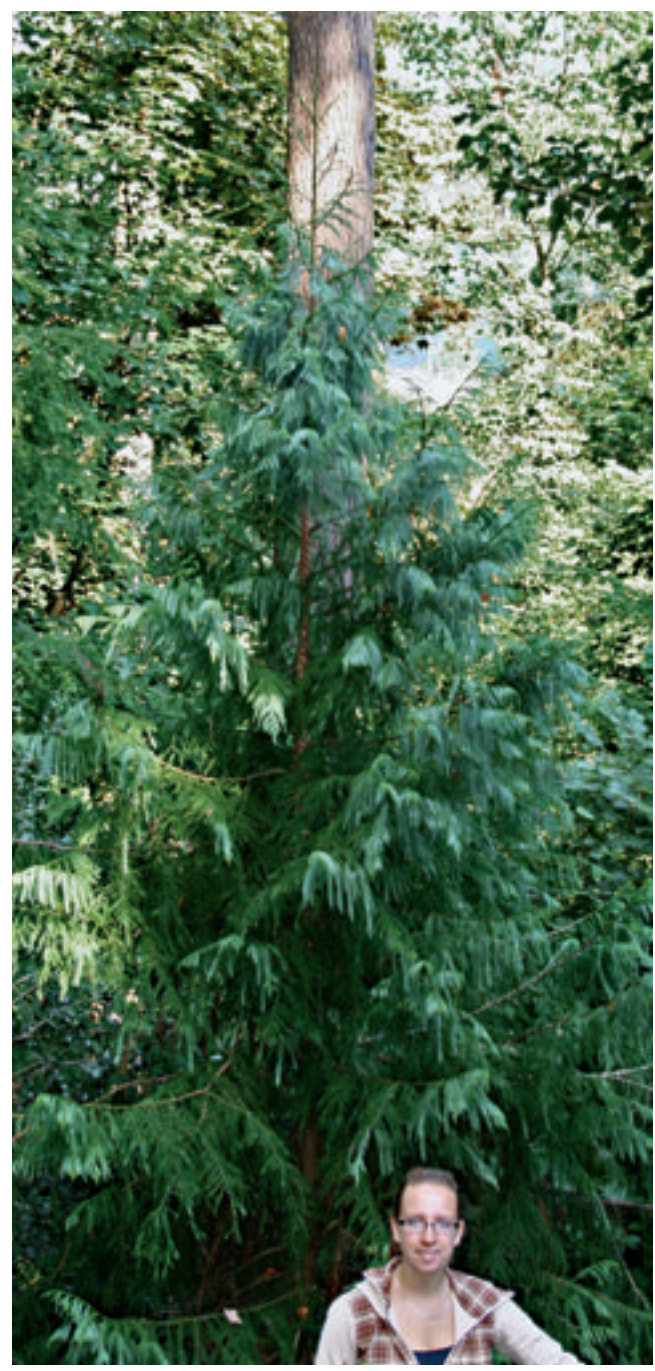

sonnigen Stelle im Altbaumbestand des Gartenarboretums ausgepflanzt. Die Pflanze stammt aus Taiwan und wurde 1996 als Saat vom Taiwan Forestry Research Institute gezogen; das Saatgut stammt aus einer Höhe von $1800 \mathrm{~m}$. Durch den umgebenden Altbaumbestand ist das Exemplar gut vor Wintersonne und austrocknenden Winden geschützt. Der Baum erwies sich als vollkommen robust und winterhart und hat selbst im Winter 2008/2009 mit den lang anhaltenden Frostperioden und Tiefsttemperaturen von minus $15^{\circ} \mathrm{C}$ keinerlei Schäden aufgewiesen. Er ist inzwischen $280 \mathrm{~cm}$ hoch, bei einem terminalen Jahreszuwachs von über $40 \mathrm{~cm}$.

Neben den klimatischen Ansprüchen der Taiwanie ist die Substratwahl im Freiland ein wichtiger Faktor, der zum Kulturerfolg führt. Die Art liebt feuchte, leicht saure und gut dränierte Böden (HuxLey 1992). Die Offenporigkeit und Drainage des Substrates wird in Bonn durch großzügige Lava oder Bimszugabe erhöht.

Da uns in Deutschland nur Exemplare bekannt sind, die ab den 1980er-Jahren im Freiland ausgepflanzt wurden - und dies wahrscheinlich nur unvollständig - sind die Autoren sehr an Informationen zu weiteren Pflanzstandorten und den hiermit verbundenen Erfahrungen interessiert.

\section{Literatur}

DALIMORE, W. 1937: A handbook of Coniferaceae and Ginkgoaceae. 4. Aufl. - London.

DÖRKEN, V. \& HÖGgemeier, A. 2009:

Botanisch-dendrologische Streifzüge. - Bochum. ENCKE, F. 1958: Pareys Blumengärtnerei,

Bd. 1. - Hamburg.

FARJON, A. 2005: A monograph of Cupressaceae and Sciadopitys. - Kew.

FARJON, A. 2008: A natural history of Conifers. - London. GrimshaW, J. \& BAYTON, R. 2009: New trees.

Recent introductions to cultivation. - Chicago.

Huxley, A. 1992: The New Royal Horticultural Society. Dictionary of Gardening, Bd. 4. - London.

TARouca, E. S. \& SCHNEIDER, C. 1923: Unsere Freilandnadelgehölze. - Wien.

Abb. 1: Taiwania im Arboretum Forstamt Freiburg Günterstal. 